Volume 135, Number 11, November 2007, Pages 3641-3648

S 0002-9939(07)09057-0

Article electronically published on August 6, 2007

\title{
SUB- AND SUPERADDITIVE PROPERTIES OF EULER'S GAMMA FUNCTION
}

\author{
HORST ALZER
}

(Communicated by Andreas Seeger)

Abstract. Let $\alpha>0$ and $0<c \neq 1$ be real numbers. The inequality

$$
\left(\frac{\Gamma(x+y+c)}{\Gamma(x+y)}\right)^{1 / \alpha}<\left(\frac{\Gamma(x+c)}{\Gamma(x)}\right)^{1 / \alpha}+\left(\frac{\Gamma(y+c)}{\Gamma(y)}\right)^{1 / \alpha}
$$

holds for all positive real numbers $x, y$ if and only if $\alpha \geq \max (1, c)$. The reverse inequality is valid for all $x, y>0$ if and only if $\alpha \leq \min (1, c)$.

\section{INTRODUCTION}

A real function $F$ defined on a set $S \subset \mathbf{R}^{n}$ is called subadditive, if

$$
F(x+y) \leq F(x)+F(y)
$$

for all $x, y \in S$ with $x+y \in S$. If inequality (1.1) is strict, then $F$ is said to be strictly subadditive. Moreover, if $-F$ is subadditive, then $F$ is called superadditive. Many interesting properties of these functions can be found in the research articles [5]-[11], [13], [17], [18]-[21], [24], [26], [29].

Sub- and superadditive functions have important applications in various fields, like functional analysis and semi-group theory [16], theory of differential equations [18], theory of convex sets [22], and statistics [28]. Moreover, sub- and superadditive problems are discussed in the theory of functional inequalities [12], [27], in number theory [14], [25], in the theory of trigonometric polynomials [3], and also in the theory of special functions [2], [4], [28].

It is the aim of this paper to study sub- and superadditive properties of Euler's gamma function,

$$
\Gamma(x)=\int_{0}^{\infty} e^{-t} t^{x-1} d t \quad(x>0) .
$$

More precisely, we consider the function

$$
f_{\alpha, c}(x)=\left(\frac{\Gamma(x+c)}{\Gamma(x)}\right)^{1 / \alpha} \quad(\alpha>0, c>0 ; x>0) .
$$

It is our aim to determine all parameters $\tilde{\alpha}, \tilde{c}$ and $\alpha^{*}, c^{*}$ such that the inequalities

$$
f_{\tilde{\alpha}, \tilde{c}}(x+y)<f_{\tilde{\alpha}, \tilde{c}}(x)+f_{\tilde{\alpha}, \tilde{c}}(y) \quad \text { and } \quad f_{\alpha^{*}, c^{*}}(x)+f_{\alpha^{*}, c^{*}}(y)<f_{\alpha^{*}, c^{*}}(x+y)
$$

Received by the editors September 5, 2006.

2000 Mathematics Subject Classification. Primary 33B15, 39B62; Secondary 26D15.

Key words and phrases. Gamma and psi functions, sub- and superadditive, convex, inequalities. 
are valid for all $x, y>0$. To solve this problem we need some lemmas, which we collect in the next section. Our main result is presented in Section 3.

In the recent past, several remarkable inequalities involving the gamma function and its relatives were published. An excellent account on this subject with a detailed bibliography is given in the survey paper [15].

\section{LEMMAS}

The psi (or digamma) function is defined by

$$
\psi(x)=(\log \Gamma(x))^{\prime}=\frac{\Gamma^{\prime}(x)}{\Gamma(x)} .
$$

The following facts can be found in [1, Chapter 6].

Lemma 1. Let $n \geq 1$ be an integer, $x>0$ a real number, and $\gamma=0.5772 \ldots$ Euler's constant. Then we have

$$
\psi(x)=-\gamma+\int_{0}^{\infty} \frac{e^{-t}-e^{-x t}}{1-e^{-t}} d t \quad \text { and } \quad \psi^{(n)}(x)=(-1)^{n+1} \int_{0}^{\infty} e^{-x t} \frac{t^{n}}{1-e^{-t}} d t .
$$

Lemma 2. We have for $x \rightarrow \infty$ :

$$
\begin{gathered}
x^{b-a} \frac{\Gamma(x+a)}{\Gamma(x+b)} \sim 1+\frac{(a-b)(a+b-1)}{2 x}+\cdots, \\
\psi(x) \sim \log x-\frac{1}{2 x}-\frac{1}{12 x^{2}}+\cdots, \\
\psi^{(n)}(x) \sim(-1)^{n+1}\left[\frac{(n-1) !}{x^{n}}+\frac{n !}{2 x^{n+1}}+\cdots\right] \quad(n=1,2, \ldots) .
\end{gathered}
$$

Lemma 3. Let $n \geq 0$ be an integer and $x>0$ a real number. Then we have

$$
\psi^{(n)}(x+1)=\psi^{(n)}(x)+(-1)^{n} \frac{n !}{x^{n+1}} .
$$

The next result is due to Petrović [23, pp. 22-23].

Lemma 4. Let $f:[0, \infty) \rightarrow \mathbf{R}$ be strictly convex. Then we have for $x, y>0$ :

$$
f(x)+f(y)<f(x+y)+f(0) .
$$

If $f$ is strictly concave, then the reverse inequality holds.

We also need an elementary monotonicity lemma.

Lemma 5. Let $r \in(0,1)$ and

$$
G_{r}(u, c)=\frac{\left(1-r^{c(1+u)}\right)\left(1-r^{c(1-u)}\right)}{\left(1-r^{1+u}\right)\left(1-r^{1-u}\right)} .
$$

If $0<c<1$, then $u \mapsto G_{r}(u, c)$ is strictly decreasing on $(0,1)$. And, if $c>1$, then $u \mapsto G_{r}(u, c)$ is strictly increasing on $(0,1)$.

Proof. Let $u \in(0,1), c>0$, and $H_{r}(u, c)=\log G_{r}(u, c)$. Partial differentiation gives

$$
\frac{\partial}{\partial u} H_{r}(u, c)=(\log r)\left[\frac{c}{r^{-c(1-u)}-1}-\frac{c}{r^{-c(1+u)}-1}-\frac{1}{r^{-(1-u)}-1}+\frac{1}{r^{-(1+u)}-1}\right]
$$


and

$$
\frac{\partial^{2}}{\partial u \partial c} H_{r}(u, c)=(\log r)[p(y)-p(z)]
$$

where

$$
p(x)=\frac{1}{x-1}-\frac{x \log x}{(x-1)^{2}}, \quad y=r^{-c(1-u)}, \quad z=r^{-c(1+u)} .
$$

Let $x>1$ and

$$
q(x)=\frac{(x-1)^{3}}{x+1} p^{\prime}(x)=\log x-\frac{2(x-1)}{x+1} .
$$

We have

$$
q^{\prime}(x)=\frac{1}{x}\left(\frac{x-1}{x+1}\right)^{2}>0 \quad \text { and } \quad q(1)=0 .
$$

This implies that $p$ is strictly increasing on $(1, \infty)$. Since $1<y<z$, we obtain $p(y)<p(z)$, so that $(2.7)$ leads to

$$
\frac{\partial^{2}}{\partial u \partial c} H_{r}(u, c)>0
$$

It follows that $c \mapsto(\partial / \partial u) H_{r}(u, c)$ is strictly increasing on $(0, \infty)$ with $(\partial / \partial u) H_{r}(u, 1)=0$. This implies that $(\partial / \partial u) H_{r}(u, c)$ is negative if $0<c<1$, $0<u<1$, and that $(\partial / \partial u) H_{r}(u, c)$ is positive if $c>1,0<u<1$.

The final two lemmas provide properties of functions, which are defined in terms of $\psi$ and $\psi^{\prime}$.

Lemma 6. Let

$$
\phi_{c}(x)=\frac{\psi^{\prime}(x)-\psi^{\prime}(x+c)}{[\psi(x+c)-\psi(x)]^{2}} .
$$

If $0<c<1$, then $\phi_{c}$ is strictly increasing on $(0, \infty)$. And, if $c>1$, then $\phi_{c}$ is strictly decreasing on $(0, \infty)$.

Proof. From Lemma 1 we conclude that $\phi_{c}(x)$ is positive for $x, c>0$. We define

$$
g_{c}(x)=\log \phi_{c}(x) .
$$

Differentiation yields

$$
\left[\psi^{\prime}(x)-\psi^{\prime}(x+c)\right][\psi(x+c)-\psi(x)] g_{c}^{\prime}(x)
$$

$$
=[\psi(x+c)-\psi(x)]\left[\psi^{\prime \prime}(x)-\psi^{\prime \prime}(x+c)\right]+2\left[\psi^{\prime}(x)-\psi^{\prime}(x+c)\right]^{2}=h_{c}(x), \quad \text { say. }
$$

Using (2.1) we obtain the integral representations

$$
\begin{gathered}
\psi(x+c)-\psi(x)=\int_{0}^{\infty} e^{-x t} \Delta_{c}(t) d t, \\
\psi^{\prime}(x+c)-\psi^{\prime}(x)=-\int_{0}^{\infty} e^{-x t} t \Delta_{c}(t) d t,
\end{gathered}
$$

and

$$
\psi^{\prime \prime}(x+c)-\psi^{\prime \prime}(x)=\int_{0}^{\infty} e^{-x t} t^{2} \Delta_{c}(t) d t
$$

with

$$
\Delta_{c}(t)=\frac{1-e^{-c t}}{1-e^{-t}}
$$


Next, we apply the convolution theorem for Laplace transforms. We get

$$
\begin{aligned}
h_{c}(x)= & -\int_{0}^{\infty} e^{-x t} \Delta_{c}(t) d t \int_{0}^{\infty} e^{-x t} t^{2} \Delta_{c}(t) d t+2\left(\int_{0}^{\infty} e^{-x t} t \Delta_{c}(t) d t\right)^{2} \\
= & -\int_{0}^{\infty} e^{-x t} \int_{0}^{t} s^{2} \Delta_{c}(s) \Delta_{c}(t-s) d s d t \\
& \quad+2 \int_{0}^{\infty} e^{-x t} \int_{0}^{t} s(t-s) \Delta_{c}(s) \Delta_{c}(t-s) d s d t \\
= & \int_{0}^{\infty} e^{-x t} I_{c}(t) d t
\end{aligned}
$$

where

$$
I_{c}(t)=\int_{0}^{t} s(2 t-3 s) \Delta_{c}(s) \Delta_{c}(t-s) d s \quad(t>0) .
$$

The substitution $s=t(1+u) / 2$ yields

$$
\begin{aligned}
(2 / t)^{3} I_{c}(t) & =\int_{-1}^{1}\left(1-2 u-3 u^{2}\right) \Delta_{c}(t(1+u) / 2) \Delta_{c}(t(1-u) / 2) d u \\
& =\int_{-1}^{1}\left(1-3 u^{2}\right) \Delta_{c}(t(1+u) / 2) \Delta_{c}(t(1-u) / 2) d u \\
& =2 \int_{0}^{1}\left(1-3 u^{2}\right) \Delta_{c}(t(1+u) / 2) \Delta_{c}(t(1-u) / 2) d u \\
& =2 \int_{0}^{1}\left(1-3 u^{2}\right) G_{r}(u, c) d s,
\end{aligned}
$$

where $G_{r}(u, c)$ is defined in (2.6) and $r=e^{-t / 2} \in(0,1)$. We distinguish two cases.

Case 1. $0<c<1$.

Applying Lemma 5 gives for $0<u<1, u \neq 1 / \sqrt{3}$ :

$$
\left(1-3 u^{2}\right) G_{r}(u, c)>\left(1-3 u^{2}\right) G_{r}(1 / \sqrt{3}, c) .
$$

This leads to

$$
\int_{0}^{1}\left(1-3 u^{2}\right) G_{r}(u, c) d u>G_{r}(1 / \sqrt{3}, c) \int_{0}^{1}\left(1-3 u^{2}\right) d u=0 .
$$

Combining (2.14)-(2.16) we conclude that $h_{c}(x)>0$ for $x>0$. It follows from (2.9) that $g_{c}$ is strictly increasing on $(0, \infty)$.

Case 2. $c>1$.

Lemma 5 yields for $0<u<1, u \neq 1 / \sqrt{3}$ :

$$
\left(1-3 u^{2}\right) G_{r}(u, c)<\left(1-3 u^{2}\right) G_{r}(1 / \sqrt{3}, c),
$$

which implies

$$
\int_{0}^{1}\left(1-3 u^{2}\right) G_{r}(u, c) d u<G_{r}(1 / \sqrt{3}, c) \int_{0}^{1}\left(1-3 u^{2}\right) d u=0 .
$$

From $(2.9),(2.14),(2.15)$, and $(2.17)$ we obtain that $g_{c}$ is strictly decreasing on $(0, \infty)$. 
Lemma 7. Let $x>0$ and

$$
\delta_{c}(x)=\psi^{\prime}(x)-\psi^{\prime}(x+c)-[\psi(x)-\psi(x+c)]^{2} .
$$

If $0<c<1$, then $\delta_{c}(x)>0$. And, if $c>1$, then $\delta_{c}(x)<0$.

Proof. First, we assume that $0<c<1$. Using (2.10)-(2.13) we obtain for $x>0$ :

$\delta_{c}^{\prime}(x)=\psi^{\prime \prime}(x)-\psi^{\prime \prime}(x+c)-2[\psi(x)-\psi(x+c)]\left[\psi^{\prime}(x)-\psi^{\prime}(x+c)\right]=\int_{0}^{\infty} e^{-x t} J_{c}(t) d t$

with

$$
J_{c}(t)=2 \int_{0}^{t} s \Delta_{c}(s) \Delta_{c}(t-s) d s-t^{2} \Delta_{c}(t) \quad(t>0) .
$$

The substitution $s=t(1+u) / 2$ leads to

$$
\left(1 / t^{2}\right) J_{c}(t)=\int_{0}^{1} \Delta_{c}(t(1+u) / 2) \Delta_{c}(t(1-u) / 2) d u-\Delta_{c}(t)=\int_{0}^{1}\left[G_{r}(u, c)-\Delta_{c}(t)\right] d u,
$$

where $G_{r}(u, c)$ is defined in (2.6) and $r=e^{-t / 2} \in(0,1)$. Applying Lemma 5 gives for $u \in(0,1)$ and $t>0$ :

$$
G_{r}(u, c)<G_{r}(0, c)=\left(\frac{1-e^{-c t / 2}}{1-e^{-t / 2}}\right)^{2}<\frac{1-e^{-c t}}{1-e^{-t}}=\Delta_{c}(t) .
$$

From (2.19) and (2.20) we conclude that $J_{c}$ is negative on $(0, \infty)$, so that $(2.18)$ implies that $\delta_{c}$ is strictly decreasing on $(0, \infty)$. Applying (2.3) and (2.4) yields

$$
\lim _{x \rightarrow \infty} \delta_{a}(x)=0 \quad(a>0) .
$$

This leads to $\delta_{c}(x)>0$ for $x>0$.

If $c>1$, then we obtain $(2.20)$ with ' $>$ ' instead of ' $<$ '. This implies that $J_{c}$ is positive on $(0, \infty)$. It follows from $(2.18)$ and $(2.21)$ that $\delta_{c}(x)<0$ for $x>0$.

\section{MAin RESUlt}

The following convexity theorem might be of independent interest. Moreover, it is an important tool in the proof of our main result, given in Theorem 2 .

Theorem 1. Let $f_{\alpha, c}$ be the function defined in (1.2), where $\alpha>0$ and $0<c \neq 1$. We have $f_{\alpha, c}^{\prime \prime}(x)>0$ for all $x>0$ if and only if $\alpha \leq \min (1, c)$. Moreover, $f_{\alpha, c}^{\prime \prime}(x)<$ 0 holds for all $x>0$ if and only if $\alpha \geq \max (1, c)$.

Proof. We have

$$
-\alpha^{2} \frac{f_{\alpha, c}^{\prime \prime}(x)}{f_{\alpha, c}(x)}=\mu_{\alpha, c}(x)-\nu_{c}(x)
$$

where

$$
\mu_{\alpha, c}(x)=\alpha\left[\psi^{\prime}(x)-\psi^{\prime}(x+c)\right] \quad \text { and } \quad \nu_{c}(x)=[\psi(x)-\psi(x+c)]^{2} .
$$

We consider two cases.

Case 1. $\alpha \leq \min (1, c)$.

We distinguish two subcases.

Case 1.1. $\min (1, c)=1$.

Then we obtain $\alpha \leq 1<c$. Applying Lemma 7 gives

$$
\mu_{\alpha, c}(x)-\nu_{c}(x) \leq \psi^{\prime}(x)-\psi^{\prime}(x+c)-[\psi(x)-\psi(x+c)]^{2}=\delta_{c}(x)<0 .
$$

From (3.1) and (3.2) we get $f_{a, c}^{\prime \prime}(x)>0$ for $x>0$. 
Case 1.2. $\min (1, c)=c$.

Then, $\alpha \leq c<1$. Let

$$
\sigma_{\alpha, c}(x)=\log \mu_{\alpha, c}(x)-\log \nu_{c}(x) .
$$

Then we have

$$
\sigma_{\alpha, c}(x)=\log \alpha+\log \phi_{c}(x),
$$

where $\phi_{c}$ is defined in (2.8). It follows from Lemma 6 that $\sigma_{a, c}$ is strictly increasing on $(0, \infty)$. Applying $(2.3)$ and $(2.4)$ we obtain the limit relations

$$
\lim _{x \rightarrow \infty} x[\psi(x+c)-\psi(x)]=\lim _{x \rightarrow \infty} x^{2}\left[\psi^{\prime}(x)-\psi^{\prime}(x+c)\right]=c .
$$

This leads to

$$
\lim _{x \rightarrow \infty} \sigma_{\alpha, c}(x)=\lim _{x \rightarrow \infty} \log \frac{\alpha x^{2}\left[\psi^{\prime}(x)-\psi^{\prime}(x+c)\right]}{(x[\psi(x+c)-\psi(x)])^{2}}=\log (\alpha / c) \leq 0 .
$$

Thus, $\sigma_{\alpha, c}$ is negative on $(0, \infty)$. From $(3.1)$ and $(3.3)$ we conclude that $f_{a, c}^{\prime \prime}$ is positive on $(0, \infty)$.

Case 2. $\alpha \geq \max (1, c)$.

Again, we consider two subcases.

Case 2.1. $\max (1, c)=1$.

We have $c<1 \leq \alpha$. It follows from Lemma 7 that

$$
\mu_{\alpha, c}(x)-\nu_{c}(x) \geq \psi^{\prime}(x)-\psi^{\prime}(x+c)-[\psi(x)-\psi(x+c)]^{2}=\delta_{c}(x)>0 .
$$

Applying (3.1) and (3.5) we obtain $f_{\alpha, c}^{\prime \prime}(x)<0$.

Case 2.2. $\max (1, c)=c$.

Then, $1<c \leq \alpha$. Using Lemma 6 gives that $\sigma_{\alpha, c}$ is strictly decreasing on $(0, \infty)$ with

$$
\lim _{x \rightarrow \infty} \sigma_{\alpha, c}(x)=\log (\alpha / c) \geq 0 .
$$

This implies that $\sigma_{\alpha, c}(x)>0$ for $x>0$, so we obtain that $f_{\alpha, c}^{\prime \prime}$ is negative on $(0, \infty)$.

It remains to show: if $f_{\alpha, c}^{\prime \prime}(x)>0$ for $x>0$, then $\alpha \leq \min (1, c)$. And, if $f_{\alpha, c}^{\prime \prime}(x)<0$ for $x>0$, then $\alpha \geq \max (1, c)$. First, we assume that $f_{\alpha, c}^{\prime \prime}$ is positive on $(0, \infty)$. Then we get

$$
(x[\psi(x+c)-\psi(x)])^{2}>\alpha x^{2}\left[\psi^{\prime}(x)-\psi^{\prime}(x+c)\right] .
$$

Using the recurrence relation (2.5) with $n=0$ and $n=1$ we conclude that (3.6) is equivalent to

$$
(x[\psi(x+c)-\psi(x+1)]+1)^{2}>\alpha+\alpha x^{2}\left[\psi^{\prime}(x+1)-\psi^{\prime}(x+c)\right] .
$$

We let $x$ tend to $\infty$ and apply (3.4). Then (3.6) leads to $c \geq \alpha$. Next, we let $x$ tend to 0 . Then (3.7) yields $1 \geq \alpha$. Hence, $\alpha \leq \min (1, c)$. If $f_{\alpha, c}^{\prime \prime}(x)<0$ for $x>0$, then we obtain (3.6) and (3.7) with ' $<$ ' instead of ' $>$ '. This gives $c \leq \alpha$ and $1 \leq \alpha$. Thus, $\alpha \geq \max (1, c)$.

We are now in a position to determine all positive parameters $\tilde{\alpha}, \tilde{c}$ and $\alpha^{*}, c^{*}$ in (1.3).

Theorem 2. Let $\alpha>0$ and $0<c \neq 1$ be real numbers. The inequality

$$
\left(\frac{\Gamma(x+y+c)}{\Gamma(x+y)}\right)^{1 / \alpha}<\left(\frac{\Gamma(x+c)}{\Gamma(x)}\right)^{1 / \alpha}+\left(\frac{\Gamma(y+c)}{\Gamma(y)}\right)^{1 / \alpha}
$$


holds for all positive real numbers $x, y$ if and only if $\alpha \geq \max (1, c)$. The reverse inequality is valid for all $x, y>0$ if and only if $\alpha \leq \min (1, c)$.

Proof. Let $f_{\alpha, c}$ be the function defined in (1.2). We have

$$
\lim _{x \rightarrow 0} f_{\alpha, c}(x)=0 .
$$

If $\alpha \geq \max (1, c)$, then Theorem 1 reveals that $f_{\alpha, c}$ is strictly concave on $[0, \infty)$. Applying Lemma 4 leads to (3.8). If $\alpha \leq \min (1, c)$, then $f_{\alpha, c}$ is strictly convex and we obtain the reverse of (3.8).

Next, we assume that (3.8) is valid for all $x, y>0$. Setting $x=y$ gives

$$
f_{\alpha, c}(2 x)<2 f_{\alpha, c}(x) \text {. }
$$

This leads to

$$
2^{c} \cdot x^{c} \frac{\Gamma(x)}{\Gamma(x+c)} \cdot(2 x)^{-c} \frac{\Gamma(2 x+c)}{\Gamma(2 x)}<2^{\alpha}
$$

and

$$
2 \cdot \frac{\Gamma(x+1)}{\Gamma(2 x+1)} \cdot \frac{\Gamma(2 x+c)}{\Gamma(x+c)}<2^{\alpha} .
$$

We let $x$ tend to $\infty$ and apply (2.2). Then, (3.9) gives $c \leq \alpha$. Next, we let $x$ tend to 0 . Then, (3.10) yields $1 \leq \alpha$. Thus, $\alpha \geq \max (1, c)$. If the reverse of (3.8) holds for all $x, y>0$, then we get (3.9) and (3.10) with ' $>$ ' instead of ' $<$ '. This leads to $c \geq \alpha$ and $1 \geq \alpha$. Hence, $\alpha \leq \min (1, c)$. The proof of Theorem 2 is complete.

\section{ACKNOWLEDGMENT}

The author thanks the referee for the careful reading of the manuscript.

\section{REFERENCES}

[1] M. Abramowitz and I.A. Stegun (eds.), Handbook of Mathematical Functions with Formulas, Graphs and Mathematical Tables, Dover, New York, 1965. MR1225604 (94b:00012)

[2] H. Alzer, Inequalities for the Hurwitz zeta function, Proc. Royal Soc. Edinburgh 130A (2000), 1227-1236. MR1809101 (2001k:11167)

[3] H. Alzer and S. Koumandos, Sub- and superadditive properties of Fejér's sine polynomial, Bull. London Math. Soc. 38 (2006), 261-268. MR2214478 (2006m:42001)

[4] H. Alzer and S. Ruscheweyh, A subadditive property of the gamma function, J. Math. Anal. Appl. 285 (2003), 564-577. MR2005141 (2004i:33002)

[5] E.F. Beckenbach, Superadditivity inequalities, Pacific J. Math. 14 (1964), 421-438. MR0163996 (29:1295)

[6] E. Berz, Sublinear functions on R, Aequat. Math. 12 (1975), 200-206. MR0387862 (52:8700)

[7] A. Bruckner, Minimal superadditive extensions of superadditive functions, Pacific J. Math. 10 (1960), 1155-1162. MR0122943 (23:A275)

[8] A.M. Bruckner, Tests for the superadditivity of functions, Proc. Amer. Math. Soc. 13 (1962), 126-130. MR0133411 (24:A3245)

[9] A.M. Bruckner, Some relationships between locally superadditive functions and convex functions, Proc. Amer. Math. Soc. 15 (1964), 61-65. MR0156924 (28:167)

[10] A.M. Bruckner and E. Ostrow, Some function classes related to the class of convex functions, Pacific J. Math. 12 (1962), 1203-1215. MR0148822 (26:6326)

[11] G. Buskes, The Hahn-Banach theorem-surveyed, Dissert. Math. 327 (1993), 49 pp. MR1240598 (94h:46007)

[12] R. Cooper, The converses of the Cauchy-Hölder inequality and the solutions of the inequality $g(x+y) \leq g(x)+g(y)$, Proc. London Math. Soc. 26 (2) (1927), 415-432.

[13] Z. Gajda and Z. Kominek, On separation theorems for subadditive and superadditive functionals, Studia Math. 100 (1991), 25-38. MR1130135 (93h:39003) 
[14] R. Garunkštis, On some inequalities concerning $\pi(x)$, Exper. Math. 11 (2002), 297-301. MR1959270 (2003k:11143)

[15] W. Gautschi, The incomplete gamma function since Tricomi, in: Tricomi's Ideas and Contemporary Applied Mathematics, Atti Convegni Lincei, 147, Accad. Naz. Lincei, Rome, 1998, 203-237. MR1737497 (2001g:33003)

[16] E. Hille and R.S. Phillips, Functional Analysis and Semi-Groups, Amer. Math. Soc., Coll. Publ. 31, Providence R.I., 1957. MR0089373 (19:664d)

[17] R.F. Jolly, Concerning periodic subadditive functions, Pacific J. Math. 15 (1965), 159-169. MR0176005 (31:281)

[18] E. Kamke, Über die eindeutige Bestimmtheit der Integrale von Differentialgleichungen, II. Sitzungsber. Akad. Wiss. Heidelberg, Math.-nat. Kl., 1931, no. 17, 15 pp.

[19] R.G. Laatsch, Extensions of subadditive functions, Pacific J. Math. 14 (1964), 209-215. MR0162897 (29:201)

[20] J. Matkowski, Subadditive functions and a relaxation of the homogeneity condition of seminorms, Proc. Amer. Math. Soc. 117 (1993), 991-1001. MR1113646 (93e:26002)

[21] J. Matkowski and T. Światkowski, On subadditive functions, Proc. Amer. Math. Soc. 119 (1993), 187-197. MR1176072 (93k:26002)

[22] H. Minkowski, Geometrie der Zahlen, Teubner, Berlin-Leipzig, 1910.

[23] D.S. Mitrinović, Analytic Inequalities, Springer, New York, 1970. MR0274686 (43:448)

[24] L.P. Østerdal, Subadditive functions and their (pseudo-)inverses, J. Math. Anal. Appl. 317 (2006), 724-731. MR2209592 (2006k:26010)

[25] L. Panaitopol, Inequalities concerning the function $\pi(x)$ : applications, Acta Arith. 94 (2000), 373-381. MR1779949 (2001g:11144)

[26] R.A. Rosenbaum, Sub-additive functions, Duke Math. J. 17 (1950), 227-247. MR0036796 $(12: 164 a)$

[27] R.M. Tardiff and H.W. Austin, Convexity and a generalized Minkowski inequality, Houston J. Math. 15 (1989), 121-128. MR1071269 (91h:54012)

[28] S.Y. Trimble, J. Wells, and F.T. Wright, Superadditive functions and a statistical application, SIAM J. Math. Anal. 20 (1989), 1255-1259. MR1009357 (91a:26019)

[29] J.S.W. Wong, A note on subadditive functions, Proc. Amer. Math. Soc. 44 (1974), 106. MR0327985 (48:6327)

Morsbacher Str. 10, D-51545 WALdBRÖL, GermanY

E-mail address: alzerhorst@freenet.de 